Original paper

\title{
Acute-on-chronic liver failure: a single-centre experience
}

\author{
L'ubomír Skladaný, Daniela Janceková, Janka Vnenčáková, Svetlana Adamcová Selčanová, Natália Bystrianska, Juraj Šváč \\ Department Internal Medicine II of Slovak Medical University, F.D. Roosevelt University Hospital, Banská Bystrica, Slovak Republic
}

\begin{abstract}
Aim of the study: We set out to determine the applicability of acute-on-chronic liver failure (ACLF) diagnostic criteria and characteristics of thus defined ACLF sub-cohorts in a real-life clinical context.

Material and methods: Retrospective charts' analysis of consecutive patients hospitalized with decompensated liver disease. Inclusion criteria: acute decompensation, informed consent. Exclusion criteria: malignancy. Diagnostic tools: $1^{\text {st }}$ phase - CLIF-SOFA score calculated manually; $2^{\text {nd }}$ phase - CLIF-C ACLF score calculated at www.efclif.com.

Results: Of 432 hospitalized patients aged 52 years, $41 \%$ were female, with MELD 20,32\% patients had acute decompensation (AD); main triggers were alcoholic hepatitis (38\%), infections (26\%), and variceal bleeding (23\%). Of patients with AD, ACLF grades $0-3$ was present in $64 \%, 19 \%, 13 \%$, and $4 \%$, respectively. In hospital mortality according to final AD/ACLF grade in ACLF $0-3$ was $7.5 \%, 42 \%, 47 \%$, and $80 \%$, respectively $(p<0.0001)$.

Conclusions: Diagnosing ACLF is nowadays easy; it helps to stratify patients at admission, and refine risk stratification at day 7. The main trigger of AD/ACLF in this region is alcohol. Currently, there are no ACLF-specific treatments; however, timely intensive supportive care can influence the prognosis. Even though still elusive and controversial, the ACLF concept can help systematize management of patients admitted with acute decompensation of advanced chronic liver disease.
\end{abstract}

Key words: mortality, cirrhosis, diagnostic criteria, acute-on-chronic liver failure, advanced chronic liver disease.

\section{Address for correspondence}

Dr. Daniela Janceková, Department Internal Medicine II of Slovak Medical University, F.D. Roosevelt University Hospital, Nám.L.Svobodu 1, 97401 Banská Bystrica, Slovak Republic, e-mail: daniela.jancekova@gmail.com

\section{Introduction}

Acute-on-chronic liver failure (ACLF) is a relatively new concept defined in Europe based on the landmark CANONIC study as the acute decompensation of cirrhosis with organ failures and high short-term mortality; this was a prospective observational study from the European Association for the Study of the Liver (EASL) and the Chronic Liver Failure consortium (EASL-CLIF) [1,2]. Proponents of the syndrome agree on its main domains and unique immune-mediated pathophysiology (immunopathogenesis) [2-4] (Fig. 1). However, various groups have not come to a unifying validated description of individual domains which has led to at least 20 definitions of ACLF [4-7]. Differences between definitions of - and cohorts with - ACLF are substantial; this was one of the main reasons why some authorities questioned the very existence of ACLF [8]. Due to these inconsistencies, we will describe the domains of ACLF in more detail.

Domains defining ACLF (Fig. 1): 1. Chronic liver disease - ACLD (advanced chronic liver disease). Some definitions require cirrhosis as the defining stage of ACLD [4, 6]; others propose that chronic hepatitis without cirrhosis could as well be the underlying condition of ACLF $[5,7]$. Our database was conceived based on the CANONIC Study (with cirrhosis); however, liver biopsy was not the prerequisite for the diagnosis of cirrhosis. 2. The trigger is the acute insult changing the clinical equilibrium of ACLD from compensated to decompensated state (Table 1). Some authors suggested division of triggers into hepatic and 


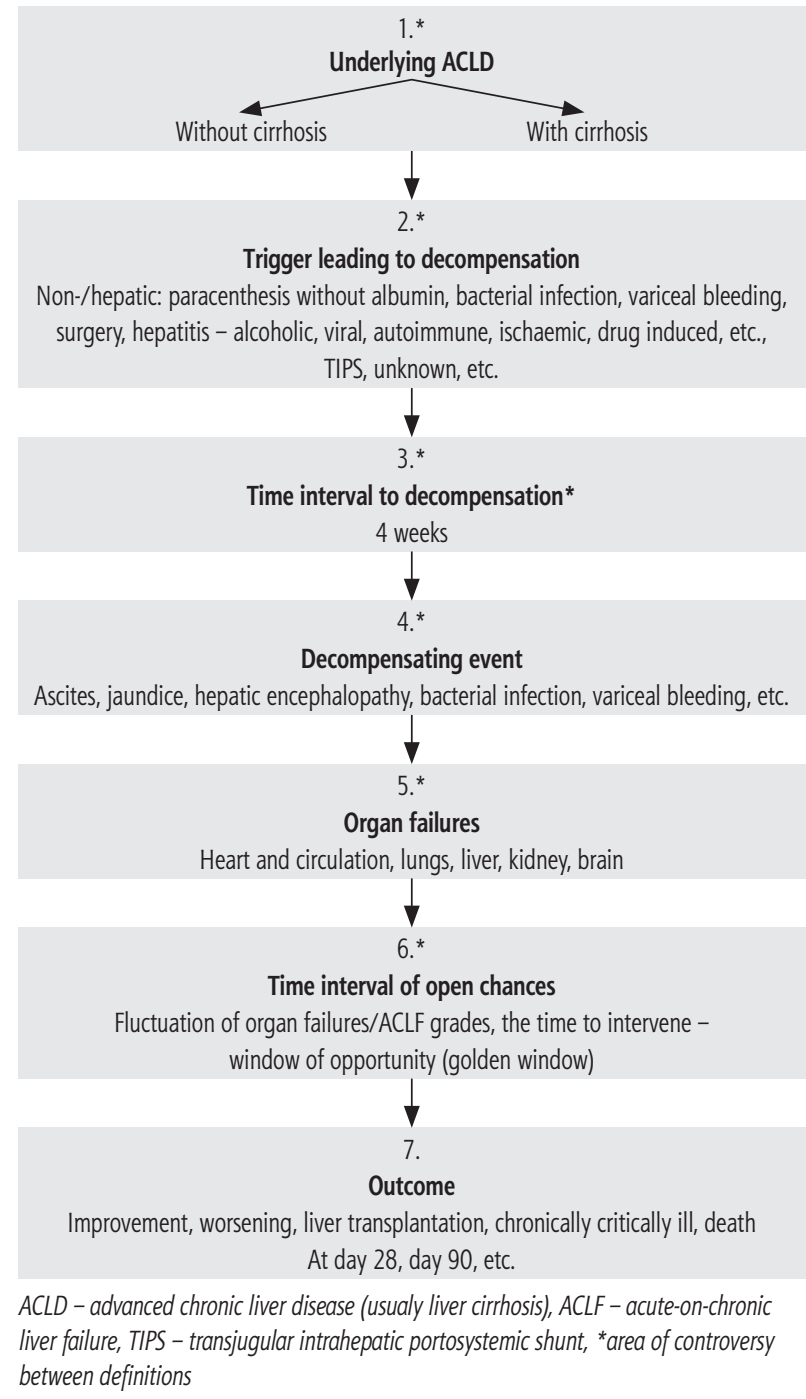

Fig. 1. Domains of the acute-on-chronic liver failure (ACLF) concept (Skladany L, 2018)

extrahepatic with the notion that they may have a different impact on prognosis $[1,9]$. The most frequent hepatic triggers of acute decompensation (AD) of ACLD are acute hepatitis - alcohol-related, viral (hepatitis A, hepatitis E, hepatitis B [flare-up, or de novo], etc.), autoimmune, ischaemic, drug-induced, etc, and extrahepatic, such as acute bacterial infections, paracentesis without albumin replacement, major surgery, transjugular intrahepatic portosystemic shunt (TIPS), variceal bleeding, and others. 3 . The event. The decompensating event is the symptom by which the decompensation of ACLD (dACLD) presents itself clinically (Table 2). The most frequent decompensating events are ascites, hepatic encephalopathy (HE), infections, and variceal bleeding. As can be seen, there is some overlap between triggers and events. 4 . Time interval 1 is the period between the event and hospital admission with the first diagnosis of AD/ACLF. Due to the
Table 1. Triggers of acute-on-chronic liver failure

\begin{tabular}{lccc}
\hline Trigger & HEGITO, 2018 & CANONIC, 2013 & SHI, 2015 \\
\hline AAH & $38 \%$ & $24.5 \%$ & $6.2 \%$ \\
\hline Bacterial infection & $26 \%$ & $32.6 \%$ & $27.9 \%$ \\
\hline Variceal bleeding & $23 \%$ & $13.2 \%$ & $8.9 \%$ \\
\hline Unknown & $7 \%$ & $43.6 \%$ & $20.5 \%$ \\
\hline Viral infection & $4 \%$ & - & $35.8 \%$ \\
\hline Others & $2 \%$ & - & - \\
\hline
\end{tabular}

$A A H$ - acute alcoholic hepatitis

Table 2. Baseline characteristics - etiology, decompensating events and triggers of acute-on-chronic liver failure (ACLF) (patients = 137)

\begin{tabular}{|c|c|}
\hline Variable & No. of patients (\%) \\
\hline Age (years) & $57(19-78)$ \\
\hline Gender female & $56(41 \%)$ \\
\hline \multicolumn{2}{|l|}{ Etiology of ACLD } \\
\hline ALD & $116(85 \%)$ \\
\hline Autoimmune syndromes & $13(9 \%)$ \\
\hline Viral hepatitis B + C & $5(4 \%)$ \\
\hline NAFLD & $3(2 \%)$ \\
\hline MELD (median) & $20(6-50)$ \\
\hline Child-Pugh score (median) & C10 (A5-C14) \\
\hline ACLF 0 (pure AD) & $87(64 \%)$ \\
\hline ACLF 1 ( $\%$ of all AD/\% of ACLF) & $26(19 \% / 52 \%)$ \\
\hline ACLF 2 & $18(13 \% / 36 \%)$ \\
\hline ACLF 3 & $6(4 \% / 12 \%)$ \\
\hline \multicolumn{2}{|l|}{ Decompensating events } \\
\hline Ascites & $66(48 \%)$ \\
\hline Upper-Gl bleeding & $36(27 \%)$ \\
\hline Hepatic encephalopathy & $18(13 \%)$ \\
\hline Infections & $17(12 \%)$ \\
\hline \multicolumn{2}{|l|}{ Triggers } \\
\hline Acute alcoholic hepatitis & $52(38 \%)$ \\
\hline Bacterial infections & $35(26 \%)$ \\
\hline Gl bleeding & $31(23 \%)$ \\
\hline Unknown & $10(7 \%)$ \\
\hline Viral hepatitis & $6(4 \%)$ \\
\hline Other - TIPS, dehydration & $3(2 \%)$ \\
\hline iACLF & $41(30 \%)$ \\
\hline $1 \mathrm{SBP}$ & $15(37 \%)$ \\
\hline 2 UTI & $10(24 \%)$ \\
\hline 3 Others & $9(21 \%)$ \\
\hline 4 Respiratory & $7(18 \%)$ \\
\hline
\end{tabular}

$A L D$ - alcohol liver disease, NAFLD - nonalcoholic fatty liver disease, iACLF - ACLF associated with infection, SBP - spontaneous bacterial peritonitis, UTI - urinary tract infection 
retrospective nature of this study, we used an arbitrary interval of 4 weeks (as in the Asian Pacific Association for the Study of the Liver [APASL] definition of ACLF) in order to avoid any uncertainty as to the key event AD. Today, the accepted interval is 3 months. 5. Organ failures. Their definition was based on the CLIF-SOFA score developed specifically for the CANONIC Study and calculated manually or, later, by the online calculator issued by EASL-CLIF Consortium (Table 3, www. clifresearch.com) $[1,4]$. In short, liver failure was defined by hyperbilirubinaemia, kidney failure by acute kidney injury (AKI diagnostic criteria for patients with cirrhosis, i.e. abrupt increase in serum creatinine level), cerebral failure by West Haven criteria for HE, respiratory failure by hypoxaemia $\left(\mathrm{PaO}_{2}, \mathrm{SpO}_{2}\right)$, circulatory failure by mean arterial pressure (MAP), and coagulation failure by the international normalized ratio (INR) [10]. Therefore, a diagnostic conclusion at admission (Day 0 - D0) would be either "pure" AD (synonymous with no ACLF, or ACLF 0), or ACLF, with grades from 1 to 3 . The mortality correlates with ACLF severity (Table 4) [4]. 6. Time interval 2 (window of opportunity, golden window) is the time between the first, and the so-called final diagnosis of $\mathrm{AD} / \mathrm{ACLF}$, which has been set at day 7 (D7); acknowledging that the syndrome in this particular phase of evolution has several important consequences: 6a. Volatility. ACLF during this period is very dynamic, and in more than $81 \%$ of patients the grade will change; in the meantime, it provides the hope that intervention could tilt the balance to improvement [10]. 6b. Prognostic restratification/personalized medicine. The dynamic nature of ACLF during the first week or so notwithstanding, recalculation of ACLF grade at D7 allows subdivision of patients into groups with different prognoses. Progressors (increase in ACLF grade, comprising around $50 \%$ of ACLF patients), Keepers (grade unchanged, $45 \%$ ), and Regressors (grade decreased, 5\%); severe early course (SEC), defined as ACLF grade $>1$ at D7, is yet another subgroup whose mortality is threefold as compared to patients without SEC [10]. 6c. Opportunity for intervention. As already mentioned, this interval seems to be the best time for an intervention either with the current best supportive care or for more specific treatments under investigation [11-15]. 7. The outcome has been scrutinized as the most im-

Table 3. European Association for the Study of Liver-Chronic Liver failure organ failures definition - CLIF-SOFA Score [1, 4]

\begin{tabular}{|c|c|c|c|}
\hline Organ system & Score $=1$ & Score $=2$ & Score $=3$ \\
\hline Liver (mg/dl) & Bilirubin $<6$ & $6 \leqslant$ Bilirubin $\leqslant 12$ & Bilirubin $>12$ \\
\hline Kidney (mg/dl) & Creatinine $<2$ & Creatinine $\geqslant 2<3.5$ & Creatinine $\geqslant 3.5$ or renal replacement \\
\hline Brain (West-Haven) & Grade 0 & Grade 1-2 & Grade 3-4 \\
\hline Coagulation & INR $<2.0$ & $2.0 \leqslant \operatorname{INR}<2.5$ & INR $\geqslant 2.5$ \\
\hline Circulation & $\mathrm{MAP} \geqslant 70 \mathrm{mmHg}$ & $\mathrm{MAP}<70 \mathrm{mmHg}$ & Vasopressors \\
\hline \multicolumn{4}{|l|}{ Respiratory: } \\
\hline $\mathrm{PaO}_{2} / \mathrm{FiO}_{2}$ & $>300$ & $\leqslant 300->200$ & $\leqslant 200$ \\
\hline or $\mathrm{SpO}_{2} / \mathrm{FiO}_{2}$ & $>357$ & $>214-\leqslant 357$ & $\leqslant 214$ \\
\hline
\end{tabular}

Bold areas indicate the definition of each organ failure. CLIF - chronic liver failure, FiO - fraction of inspired oxygen, INR - international normalised ratio, MAP - mean arterial pressure, $\mathrm{PaO}_{2}$ - partial pressure of arterial oxygen, RRT - renal replacement therapy, SOFA - Sequential Organ Failure Assessment, SpO - pulse oxymetric saturation

Table 4. Acute-on-chronic liver failure (ACLF) grades and mortality $[1,4]$

\begin{tabular}{|c|c|c|c|c|}
\hline & \multicolumn{4}{|c|}{ ACLF grade } \\
\hline & ACLF 0 & ACLF 1 & ACLF 2 & ACLF 3 \\
\hline Definition & $\begin{array}{l}\text { - No organ failure } \\
\text { - Single organ failure in patients with } \\
\text { a serum creatinine level of }<1.5 \mathrm{mg} / \mathrm{dl} \\
\text { and no hepatic encephalopathy } \\
\text { - Cerebral failure in patients with a serum } \\
\text { creatinine level of }<1.5 \mathrm{mg} / \mathrm{dl}\end{array}$ & $\begin{array}{l}\text { - Single kidney failure } \\
\text { - Single liver, coagulation, circulatory or } \\
\text { lung failure that is associated with a serum } \\
\text { creatinine level of } 1.5-1.9 \mathrm{mg} / \mathrm{dl} \text { and/or } \\
\text { hepatic encephalopathy grade } 1 \text { or grade } 2 \\
\text { - Single brain failure with a serum creatinine } \\
\text { level of } 1.5-1.9 \mathrm{mg} / \mathrm{dl}\end{array}$ & $\begin{array}{l}\text { Two organs } \\
\text { failures }\end{array}$ & $\begin{array}{l}\text { Three organ } \\
\text { failures or more }\end{array}$ \\
\hline $\begin{array}{l}\text { CANONIC 28-day } \\
\text { mortality }\end{array}$ & $5 \%$ & $22 \%$ & $32 \%$ & $77 \%$ \\
\hline HEGITO 28-day mortality & $5 \%$ & $33 \%$ & $33 \%$ & $80 \%$ \\
\hline
\end{tabular}


portant measure for evaluating the syndrome, its management, and for comparison of studies and cohorts. At present, there is a consensus about several endpoints: D0 ACLF grade, D7 grade, SEC, liver transplantation (LTx), and mortality - in-hospital, and at D28, and D90. Other endpoints are under investigation [10].

\section{Aim of the study}

To characterize ACLF in real-life clinical practice; to scrutinize the applicability of diagnostic tools; and to determine the prevalence of ACLF in patients admitted with dACLD, the structure of domains, demographic and clinical characteristics of patients, and outcome.

\section{Novelty statement}

To the best of the authors' knowledge, this is the first study on ACLF from real-life clinical practice in the Central European region. Evidence before this study: That AD of ACLD could be a separate entity markedly different from chronic decompensation was proposed relatively recently. Especially $\mathrm{AD}$ with organ failures (ACLF) could have different pathogenesis (immunopathogenesis) from chronic decompensation (CD), and is known to bear a much poorer shortterm prognosis. However, most of the data on ACLF have come from multicentric studies undertaken at renowned academic centres; therefore, validating the diagnostic and management principles by comparison with real-life clinical practice - especially from a limited-resources setting - is of importance.

Added value of this study: The prevalence, triggers, events, subtypes, and prognosis of AD/ACLF lend support to the notion that the syndrome can be a distinct entity with a different prognosis from the $\mathrm{CD}$. Moreover, the study confirms that the syndrome can be diagnosed bedside in real-life clinical practice.

Ethical approval: All procedures involving human participants have been approved according to the ethical standards of the institutional and/or national research committee, including the 1964 Helsinki Declaration and its later amendments or comparable ethical standards.

\section{Material and methods}

In this retrospective registry study, we reviewed the files of consecutive patients admitted to the liver unit of a regional university hospital with the diagnosis of dACLD. Study interval: July 2014 - May 2017. Inclusion criteria: We included patients 18 years of age and older who provided written informed consent and were admitted with $\mathrm{dACLD}$. We defined $\mathrm{AD}$ as the trigger-to-event interval of up to 4 weeks, which was confirmed per protocol by one of the authors (D.J.) (Fig. 1). Triggers and decompensating events were defined according to the CANONIC Study and are listed in Tables 1 and 2, respectively. One notable deviation from the CANONIC definition pertained to acute alcoholic hepatitis (AAH) in that we did not require liver biopsy for confirmation of the diagnosis (Tables 1,2) $[1,3]$. Organ failures were defined according to the CANONIC Study, at first by manually calculating the CLIF-SOFA Score, and later by the newly introduced CLIF-C online calculator (Table 3; www.clifresearch. com). Exclusion criteria: We excluded patients with hepatocellular carcinoma and those in whom we were unable to retrieve the variables necessary for diagnosing $\mathrm{AD} / \mathrm{ACLF}$. Recorded variables: For this analysis we recorded age (years - y), sex, aetiology of ACLD, trigger of $A D$, and $D 0$ and D7 variables: AD/ACLF, MELD score and its components (Model for the End-Stage Liver Disease), Child-Pugh score and its components, bacterial infections, acute kidney injury (AKI), length of hospital stay (LOS, days), and mortality.

\section{Statistical analysis}

We used a certified statistical program, MedCalc version 13.1.2. (MedCalc Software VAT registration number BE 0809344 640, Member of International Association of Statistical Computing, Ostend, Belgium). Comparisons of continuous variables between groups were carried out using parametric ( $t$-test) or non-parametric (Mann-Whitney) tests; associations between categorical variables were analysed using the $\chi^{2}$ test and Fisher's exact test, as appropriate. We considered a $p$-value $<0.05$ to be statistically significant.

\section{Results}

Of 432 patients hospitalized with dACLD during the median study interval of 35 months, 137 patients had $\mathrm{AD}$ (32\%) (Fig. 2). Their median age was 57 years (20-79), and 56 patients of them were female (41\%). The most common aetiology of ACLD was alcoholic liver disease (ALD), present in 166 patients (85\%); other aetiologies are listed in the table (Table 2). Median MELD and Child-Pugh scores at admission (D0) were 20 (6-50) and $\mathrm{C} 10$ (A5-C14), respectively. The average LOS was 17 days (6-56). Triggers of AD: acute alcoholic hepatitis - 52 patients (38\%), bacterial infections - 35 patients (26\%, ACLF associated with infection iACLF), variceal bleeding - 31 patients (23\%), unknown - 10 patients (7\%), viral hepatitis -6 patients (4\%), other 


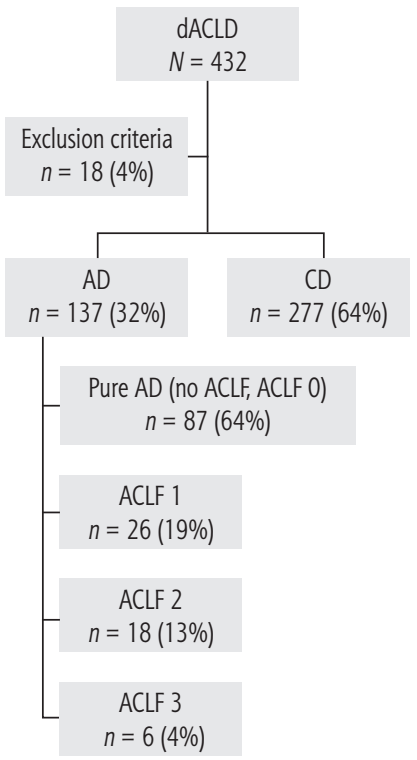

$d A C L D$ - decompensated chronic liver disease (cirrhosis), $A D$ - acute decompensation, $C D$ - chronic decompensation, $A C L F$ - acute-on-chronic liver failure

Fig. 2. Flowchart of the study

- 3 patients (2\%). Decompensating events: ascites 66 patients (48\%), variceal bleeding - 36 patients (27\%), $\mathrm{HE}-18$ patients (13\%), bacterial infection - 17 patients (12\%). "Pure" AD (no ACLF, ACLF 0), and ACLF have been diagnosed in 87, and 50 of 137 patients with $\mathrm{AD}$ (64\%, and 36\%, respectively); ACLF 1 in 26 patients (19\%), ACLF 2 - 18 patients (13\%), ACLF 3 - 6 patients (4\%) (Fig. 3). Day 7 Progressors, Keepers, Regressors, and patients with SEC represented 12 patients (9\%), 104 patients (76\%), 21 patients (15\%) and 20 patients (15\%), respectively. Of 41 patients with iACLF, sponta-

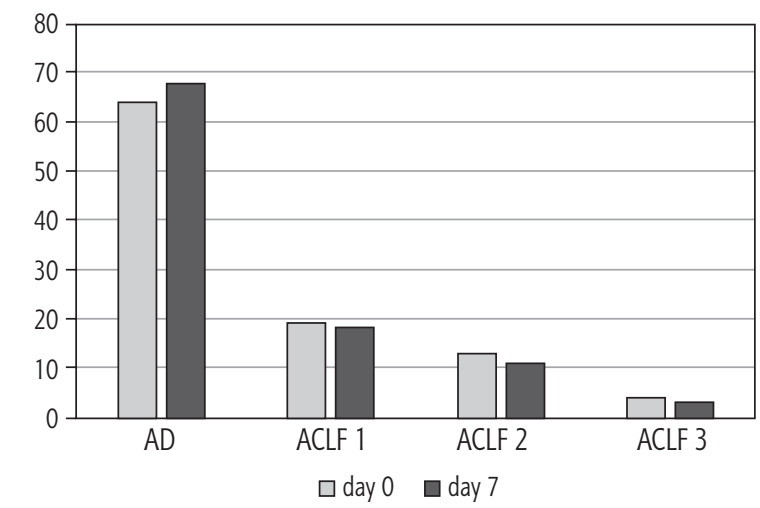

\begin{tabular}{ccccc}
\hline & AD & ACLF 1 & ACLF 2 & ACLF 3 \\
\hline Day 0 & 87 patients & 26 patients & 18 patients & 6 patients \\
& $(64 \%)$ & $(19 \%)$ & $(13 \%)$ & $(4 \%)$ \\
\hline Day 7 & 93 patients & 24 patients & 15 patients & 5 patients \\
& $(68 \%)$ & $(18 \%)$ & $(11 \%)$ & $(3 \%)$ \\
\hline
\end{tabular}

Fig. 3. Prevalence of acute decompensation/acute-on-chronic liver failure (AD/ACLF) on day 0 and day 7 neous bacterial peritonitis (SPB) represented 15 patients (37\%), urinary tract infections (UTI) 10 patients (24\%), respiratory tract infections (RTI) -7 patients $(18 \%)$, and other infections 9 patients (21\%). Median LOS in $\mathrm{AD}$ patients was 14 days (3-128), 28 patients (20\%) died during LOS. The 28-day and 90-day mortality according to final $\mathrm{AD} / \mathrm{ACLF}$ grade was as follows: Pure $\mathrm{AD}$ (ACLF 0) - 5 patients (5\%) and 5 patients (5\%), ACLF 1 -8 patients (33\%) and 10 patients (42\%), ACLF 2 -5 patients (33\%) and 5 patients (33\%), and ACLF 3 4 patients (80\%) and 4 patients $(80 \%)(p=0.0000006$, and $p=0.0000001$ ) (Fig. 4). In-hospital mortality according to final $\mathrm{AD} / \mathrm{ACLF}$ grade was $7.5 \%$ in pure $\mathrm{AD}$ group, $42 \%$ in ACLF 1 group, 47\% in ACLF 2 group and $80 \%$ in ACLF 3 group (Fig. 5). In-hospital mortality of patients with SEC, without SEC, and with mere AD was $55 \%, 42 \%$, and $7.5 \%$, respectively $(p=0.0000001$ ) (Fig. 6).

\section{Discussion}

Our study demonstrates that the applicability of diagnostic criteria for ACLF in real-life clinical practice are feasible. Diagnosing ACLF has several possible consequences: early allocation of patients to intensive care unit; early best supportive care (namely hit-hard antibiotic strategy, volume replacement with albumin, proper use of AKI criteria, LTx scrutiny, futility rules, etc.); early communication with patients and family members about the prognosis; communication with stakeholders about the economic burden of the syndrome.

Approximately one-third of all patients hospitalized with $\mathrm{dACLD}$ were diagnosed with $\mathrm{AD}$, and one-

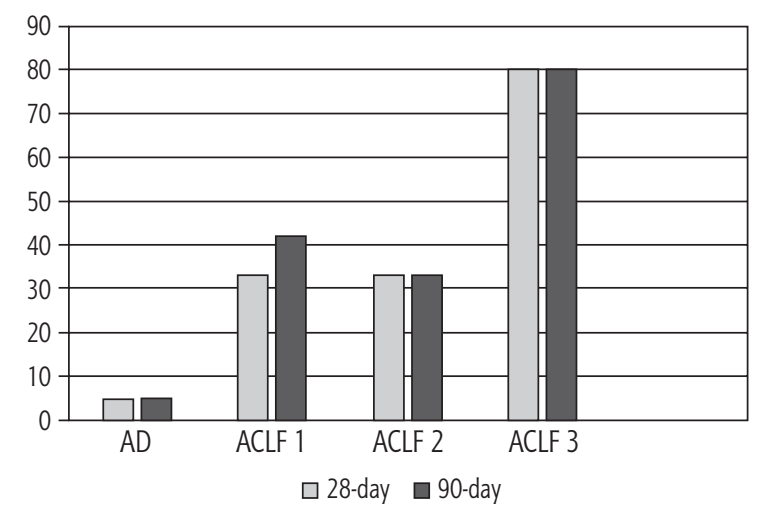

\begin{tabular}{cccccc}
\hline & AD & ACLF 1 & ACLF 2 & ACLF 3 & $p$ \\
\hline 28-day & 5 patients & 8 patients & 5 patients & 4 patients & 0.0000006 \\
& $(5 \%)$ & $(33 \%)$ & $(33 \%)$ & $(80 \%)$ & \\
\hline 90-day & 5 patients & 10 patients & 5 patients & 4 patients & 0.0000001 \\
& $(5 \%)$ & $(42 \%)$ & $(33 \%)$ & $(80 \%)$ & \\
\hline
\end{tabular}

Fig. 4. 28-day and 90-day mortality according to final acute decompensation/ acute-on-chronic liver failure (AD/ACLF) grade 


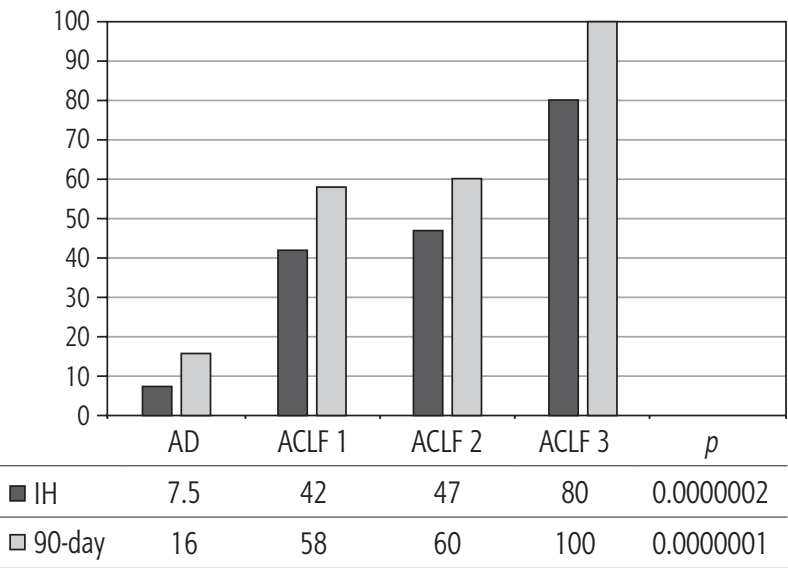

$A D$ - acute decompensation, $A C L F$ - acute-on-chronic liver failure

Fig. 5. In-hospital (IH) and 90-day mortality according to final ACLF grade (in \%)

third of AD patients had ACLF. This is at variance with $77.4 \%$ prevalence of $\mathrm{AD}$ at enrolment in the CANONIC Study, which could best be explained by the character of liver units in the CANONIC Study in comparison with ours, and explicit time interval; the other explanation - possible other differences in diagnostic criteria - is considered less probable because of their small scale [3]. The prevalence of ACLF grades in our cohort and the CANONIC Study at enrolment were similar: ACLF $1-19 \%$ and $11 \%$, ACLF $2-13 \%$ and $8 \%$, and ACLF $3-4 \%$ and $3.5 \%$, respectively; these figures are formally hard to compare considering the differences between studies. The most common underlying aetiology of ACLD in our cohort was ALD, which reflects the overall burden of ALD in the country [16]. The representation of respective triggers and events did not show any differences from what has been seen in other studies from the West, apart from the relatively low proportion of trigger "unknown" in our cohort ( $7 \%$ vs. $40 \%$ in CANONIC), for which we do not have an explanation apart from the possible differences in diagnostic criteria for ALD (Tables 1, 2) [4-7].

The prognosis of "pure" $\mathrm{AD}$ is trivial: $28 \mathrm{D}$ mortality in the CANONIC Study and our cohort was 5\%, respectively [3]. However, mortality was significantly higher already in ACLF grade 1 (33\%), and increased to $80 \%$ in ACLF 3 ( $p=0.0000006$ ) (Fig. 4$)$. The 28 -day mortality in our patients with ACLF 1 (33\%) was higher than 28D mortality in the CANONIC Study (23\%); even though we do not know if it is the reflection of true difference, it will remain the focus of our attention. As for the relationship between prognosis and the triggers, numerically the worst and best were seen in AAH and variceal bleeding, respectively, but without statistical significance $(p=0.1492)$ (Fig. 7$)$. Similar prognosis of our ACLF triggered by a bacterial infec-

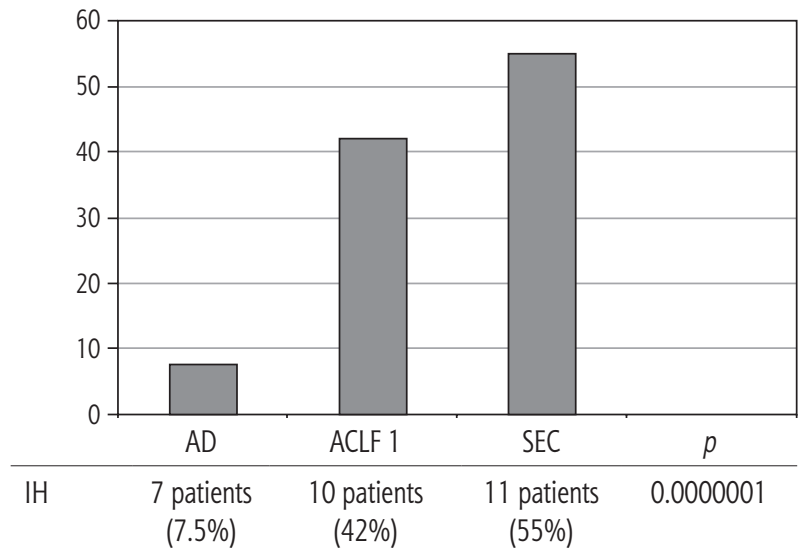

Fig. 6. In-hospital mortality according to the presence or absence of severe early course (SEC)

tion and AAH does not support the notion of different predictive potentials of hepatic vs. extrahepatic triggers $(p=0.1492)$. Alternatively, the hit to the liver by bacterial infections renders them more of a hepatic than extrahepatic nature (Fig. 7). This is in agreement with the study from Croatia where infections increased 30-day mortality 2.3-times irrespective of the degree of liver failure [17]. The most prevalent infection site was the peritoneum (spontaneous bacterial peritonitis - SBP), which, together with respective proportions of the other sites, we consider to be a real reflection of the situation in our liver unit. We do not provide the information on the types of bacteria and spectra of drug resistance, but they are under investigation [18]. In-hospital mortality in patients with SEC (55\%) was significantly higher than in patients with "pure" $\mathrm{AD}$ $(7.5 \%)$ and somewhat higher than in ACLF $1(42 \%)$ $(p=0.0000001)$ (Fig. 6). These results are in accord with the data from Gustot et al., and lend support to

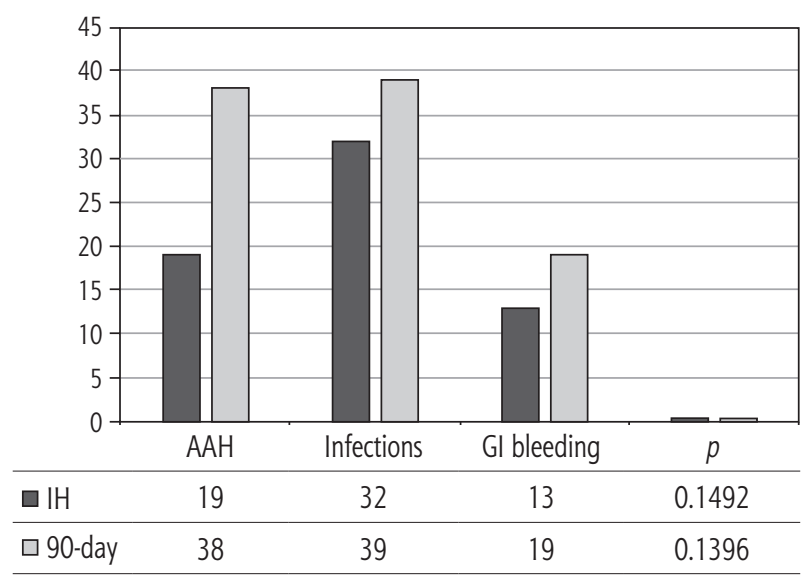

AAH - acute alcoholic hepatitis, GI-bleeding - gastrointestinal bleeding

Fig. 7. In-hospital (IH) mortality and 90-day mortality according to the trigger (in \%) 
the case for prognostic power of the dynamics of ACLF during this time interval [10]. In comparison with CANONIC, more of our patients improved over the first week; we can only speculate that this difference can be explained by the higher proportion of alcohol use as a trigger and background aetiology of ACLD, with abstinence as the causal therapy.

This study has several limitations: a retrospective, single-centre design; a relatively small number of patients; no systematic indication for liver biopsy (especially in AAH patients); no information on previous dACLD/ACLF episodes; and no follow-up data after D90 and after LTx. The main deviations from the CANONIC diagnostic protocol were as follows: introduction of 4-week interval for the diagnosis of $\mathrm{AD}$ (instead of leaving it at the discretion of attending physician); absence of liver biopsy in cases of $\mathrm{AAH}$; and not being able to identify and exclude patients on immunosuppressive drugs. On the other hand, to the best of our knowledge, this is the first study on ACLF from this geographic area; interpretation of CLIF-SOFA before the introduction of the online calculator was mastered at Paul-Brousse Hospital in Paris, and fulfilment of ACLF diagnostic criteria has been doublechecked per protocol (D.J.).

Our study does not add to the epistemological debate about the existence and meaning of ACLF; however, our work with the ACLF concept in the real-life clinical setting of the resource-limited region has helped us to re-direct adequate and timely attention to the patients at higher risk of death, which we think we would not recognize otherwise. Also, the comparison of our cohort to those from other centres is helping us in refining our diagnostic and therapeutic strategies.

\section{Conclusions}

Our study demonstrates that to diagnose ACLF in real-life clinical practice is feasible and the applicability of diagnostic tools is easy. Stratifying patients with these tools to AD and ACLF subcohorts makes sense in terms of prognosis. This stratification allows for the timely introduction of intensive and directed supportive care measures which could improve the otherwise high short-term mortality, especially if the pathophysiologically based targeted treatments currently under investigation will become available.

\section{Acknowledgments}

We are indebted to Prof. Fauzi Saliba for kindly introducing our physicians to the principles of ACLF diagnosis.

\section{Disclosure}

The authors report no conflict of interest.

\section{References}

1. Moreau R, Jalan R, Gines P, et al. Acute-on-chronic liver failure is a distinct syndrome that develops in patients with acute decompensation of cirrhosis. Gastroenterology 2013; 144: 1426-1437.

2. Albillos A, Lario M, Álvarez-Mon M, et al. Cirrhosis-associated immune dysfunction: distinctive features and clinical relevance. J Hepatol 2014; 61: 1385-1396.

3. Solà E, Ginès P. Pro: Acute-on-chronic liver failure. Liver Transpl 2017; 23: 1318-1324.

4. Arroyo V, Moreau R, Jalan R, et al. Acute-on-chronic liver failure: a new syndrome that will re-classify cirrhosis. J Hepatol 2015; 62 (1 Suppl): S131-143.

5. Sarin SK, Kedarisetty CK, Abbas Z, et al. Acute-on-chronic liver failure: consensus recommendations of the Asian Pacific Association for the Study of the Liver (APASL) 2014. Hepatol Int 2014; 8: 453-471.

6. Bajaj JS, O'Leary JG, Reddy KR, et al. Second infections independently increase mortality in hospitalized patients with cirrhosis: the North American consortium for the study of endstage liver disease (NACSELD) experience. Hepatology 2012; 56: 2328-2335.

7. Jalan R, Yurdaydin C, Bajaj JS, et al. Toward an improved definition of acute-on-chronic liver failure. Gastroenterology 2014; 147: 4-10.

8. O'Grady J. Con: Acute-on-chronic liver failure. Liver Transpl 2017; 23: 1325-1327.

9. Shi Y, Yang Y, Hu Y, et al. Acute-on-chronic liver failure precipitated by hepatic injury is distinct from that precipitated by extrahepatic insults. Hepatology 2015; 62: 232-242.

10. Gustot T, Fernandez J, Garcia E, et al. Clinical course of acuteon-chronic liver failure syndrome and effects on prognosis. Hepatology 2015; 62: 243-252.

11. Singh V, Sharma AK, Narasimhan RL, et al. Granulocyte colony-stimulating factor in severe alcoholic hepatitis: a randomized pilot study. Am J Gastroenterol 2014; 109: 1417-1423.

12. Moreau R, Rautou PE. G-CSF therapy for severe alcoholic hepatitis: targeting liver regeneration or neutrophil function? Am J Gastroenterol 2014; 109: 1424-1426.

13. Duan XZ, Liu FF, Tong JJ, et al. Granulocyte-colony stimulating factor therapy improves survival in patients with hepatitis B virus-associated acute-on-chronic liver failure. World J Gastroenterol 2013; 19: 1104-1110.

14. Kedarisetty CK, Anand L, Bhardwaj A, et al. Combination of granulocyte colony-stimulating factor and erythropoietin improves outcomes of patients with decompensated cirrhosis. Gastroenterology 2015; 148: 1362-1370.

15. Khanam, A, Trehanpati N, Sharma BC, et al. Altered frequencies of dendritic cells and IFN-gamma-secreting T cells with granulocyte colony-stimulating factor (G-CSF) therapy in acute-onchronic liver failure. Liver Int 2014; 34: 505-513.

16. Szántová M. Alkohol a pečeň pohladom hepatológa. Psychiatr Prax 2013; 14: 114-119.

17. Grgurevic I, Trkulja V, Bozin T, et al. Infection as a predictor of mortality in decompensated liver cirrhosis: exploring the relationship to severity of liver failure. Eur J Gastroenterol Hepatol 2019; doi: 10.1097/MEG.0000000000001667. 18. Fernández J, Prado V, Trebicka J, et al. Multidrug-resistant bacterial infections in patients with decompensated cirrhosis and with acute-on-chronic liver failure in Europe. J Hepatol 2019; 70: 398-411. 